\title{
Improving Photocatalytic Energy Conversion via NAD(P)H
}

Wilm Jones, ${ }^{1,5,6}$ Joseph W.H. Burnett, ${ }^{1,5}$ Jiafu Shi, ${ }^{2}$ Russell F. Howe ${ }^{*, 3}$

and Xiaodong Wang ${ }^{*, 1,4}$

${ }^{1}$ Chemical and Materials Engineering, School of Engineering, University of Aberdeen, Aberdeen AB24 3UE, United Kingdom

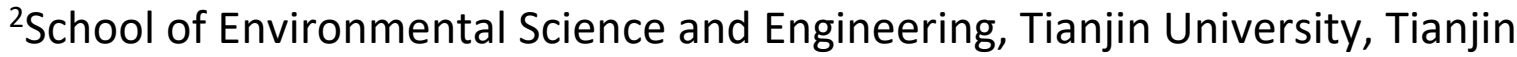
300072, China

${ }^{3}$ Department of Chemistry, University of Aberdeen, Aberdeen AB24 3UE, United Kingdom

${ }^{4}$ Chemical Engineering, Department of Engineering, Lancaster University, Lancaster LA1 4YW, United Kingdom

*Corresponding Author E-mails:

r.howe@abdn.ac.uk (RFH); xiaodong.wang@lancaster.ac.uk (XW).

${ }^{5}$ These authors contributed equally.

${ }^{6}$ Present address: Chemistry Department, University College of London, Gordon

Street, London WC1H OAJ, UK; Research Complex at Harwell, Rutherford Appleton Laboratory, Didcot OX11 OFA, UK 


\section{Abstract:}

The reduced form of nicotinamide adenine dinucleotide $(\mathrm{NAD}(\mathrm{P}) \mathrm{H})$ behaves as an energy/chemical "currency", carrying hydrogen in a biologically convertible form and donates electrons in numerous biotransformations and artificial photosynthesis. Its high cost necessitates its regeneration for reuse where photocatalysis using light energy is attractive. However, high $\mathrm{NAD}(\mathrm{P}) \mathrm{H}$ yield is only achievable via organic mediators to transfer electrons. Here, we analyse the current issues in catalytic $\mathrm{NAD}(\mathrm{P}) \mathrm{H}$ regeneration and show that a continuous-flow reactor system can realise selective $\mathrm{NAD}(\mathrm{P}) \mathrm{H}$ regeneration with $100 \%$ yield using $\mathrm{Pt} / \mathrm{C}_{3} \mathrm{~N}_{4}$ as a photocatalyst.

Keywords: NADH; Regeneration; Photocatalysis; Mediator-Free; Flow 
The prospect of exploiting solar energy in a clean, economical and inherently sustainable manner has prompted an explosion of research into photocatalysis, particularly for producing fuels or chemicals from renewable resources. One particularly attractive method for solar energy utilisation is its transformation to yield chemical energy in the form of a reducing potential such as the reduced form of nicotinamide adenine dinucleotide $(1,4-N A D(P) H) .{ }^{1} 1,4-$ $\mathrm{NAD}(\mathrm{P}) \mathrm{H}$, as a chemical/energy "currency", carries hydrogen in a biologically convertible form and donates electrons (forming $N A D(P)^{+}$consequently, see Figure 1). 1,4-NAD(P)H is a crucial component in oxygen photosynthesis and drives the Calvin-Benson-Bassham cycle for photosynthetic production of carbohydrates from $\mathrm{CO}_{2} \cdot{ }^{1}$ As a cofactor it is essential in numerous artificial biotransformations such as the conversion of $\mathrm{CO}_{2}$ to methanol, chiral synthesis and the production of valuable pharmaceuticals and fine chemicals. ${ }^{2,3}$ The high cost of $1,4-\mathrm{NAD}(\mathrm{P}) \mathrm{H}(1,4-\mathrm{NADPH} \$ 70,000 / \mathrm{mol} \text { and } 1,4-\mathrm{NADH} \$ 2,600 / \mathrm{mol})^{4}$ requires it to be regenerated..$^{2,3}$ The use of photocatalysis for this purpose (ideally using sunlight) has attracted much recent attention (see Figure 1) and paved the way for the development of artificial photosynthesis.

High yields in the photocatalytic regeneration are unfortunately only achievable using a mediator, typically an organic $\mathrm{Rh}$ complex $\left([\mathrm{Cp} * \mathrm{Rh}(\mathrm{bpy}) \mathrm{Cl}]^{+}\right)$, whose role is to selectively transfer a hydride to $N A D(P)^{+}$while the role of the photocatalyst is to regenerate the mediator. ${ }^{3,5}$ The use of a mediator is undesirable as it involves an expensive rare metal, extra preparation and product separation steps. Some literature reports exist for mediator-free systems where the enzymatically validated yield of 1,4-NAD(P)H $\left(Y_{1,4-\mathrm{NADH}}\right)$ is typically low, e.g., $<30 \%$ with byproducts $(46 \%)$ also generated using the photosensitiser tetra(4carboxyphenyl)porphyrin. ${ }^{6}$ However, often $Y_{1,4-\mathrm{NADH}}$ is not quantitatively validated, as 
summarised in Table S1. Maximising the yield and selectivity of 1,4-NAD(P)H without mediators remains a significant challenge. But it is surprising to note that the community seemingly has accepted the routine use of such mediators with little attempt made to remove them. In this work, we highlight some common features that we believe have hindered the progress of the field and likely other general photochemical processes and propose a proofof-concept flow system for mediator-free photocatalytic $N A D(P) H$ regeneration with exclusive selectivity.

A "Golden Rule" of reporting data. Firstly, scrutiny of publications on NAD(P)H regeneration shows that, surprisingly, a majority have used the UV-vis absorbance at $340 \mathrm{~nm}$ as a measure of the 1,4-NAD(P)H concentration (Figure $\mathrm{S} 1$ ). Other regeneration products which are enzymatically inactive also have UV-vis absorption in this region, such as the $1,6-\mathrm{NAD}(\mathrm{P}) \mathrm{H}$ isomer $(345 \mathrm{~nm})$ and the $(\mathrm{NAD}(\mathrm{P}))_{2}$ dimer $(\sim 340 \mathrm{~nm}) .{ }^{7}$ A successful in-situ coupling experiment with enzyme catalysis does not confirm the concentration or selectivity in the regeneration step alone. Any so-called NAD(P)H yields calculated using the molar absorption coefficient of 1,4-NAD(P)H when other molecules are produced (highly likely $3,5,6,8,9$ ), will give erroneous data. Such unvalidated data (denoted as $Y_{340} \mathrm{~nm}$ ) should be read with extra caution as they reveal little useful information.

Secondly, when the yield of 1,4-NAD(P)H is enzymatically validated (denoted as $Y_{1,4-N A D H}$ ) by an enzymatic assay (e.g., using alcohol or formate dehydrogenase), typically at the end of a reaction, curves showing a temporal increase till near $100 \%^{5,10}$ will be credible and will indicate that all $N A D(P)^{+}$is converted selectively to $1,4-N A D(P) H$. Not many studies have achieved a validated $100 \% Y_{1,4-\mathrm{NADH}}$. Many reported increasing curves cease abruptly (sometimes in only 10 mins) at well below $100 \%$ yield with no indication of what would 
happen if the reaction time was extended (Figure S1). If the yield of 1,4-NAD(P)H decreases (or levels off) at longer times instead of continuing to rise (when does its consumption begin?), the usefulness of the method reported must be questioned.

Finally, the lack of rigorous qualification and quantification of species involved ${ }^{7}$ has led to, as a third issue, the absence of some vitally important information, i.e., the conversion of the $\operatorname{NAD}(P)^{+}$reactant. A validated $Y_{1,4-\mathrm{NADH}}$ would only be useful when it reaches almost $100 \%$, otherwise the selectivity of the process is unknown. Simply confirming that the absorption at $340 \mathrm{~nm}$ is due to $1,4-\mathrm{NAD}(\mathrm{P}) \mathrm{H}$ alone and reporting $100 \%$ selectivity ignores the very real possibility of the formation of byproducts that do not absorb in this region. A further question to ask is whether the amount of $N A D(P)^{+}$consumed matches that of $1,4-N A D(P) H$ produced.

These three issues, in our opinion, prevent a deeper understanding of the reaction mechanisms and development of more efficient mediator-free (photo)catalytic systems. Much of the focus in the literature has been on fine-tuning photocatalysts for mediator regeneration, leaving the mediator-free challenge unaddressed.

"Bad" data guided understanding and design. Here we abandon the "golden rule" discussed above by presenting some conventionally considered "bad" data which led us to design a highly selective mediator-free system for photocatalytic $N A D(P) H$ regeneration. We employed graphitic carbon nitride $\left(\mathrm{g}-\mathrm{C}_{3} \mathrm{~N}_{4}\right)$ as a photocatalyst; synthesis and characterisation of the $\mathrm{g}_{-} \mathrm{C}_{3} \mathrm{~N}_{4}$ and $\mathrm{g}-\mathrm{C}_{3} \mathrm{~N}_{4}$ modified with $(3 \% \mathrm{w} / \mathrm{w})$ Pt nanoparticles are presented in the Supplemental Information (Figure S2).

Using $\mathrm{g}-\mathrm{C}_{3} \mathrm{~N}_{4}$ and $\mathrm{Pt} / \mathrm{g}-\mathrm{C}_{3} \mathrm{~N}_{4}$, we performed mediator-free photocatalytic 1,4-NADH regeneration in a static-batch reactor. Progress of the reaction was first followed by UV-Vis 
absorbance at $340 \mathrm{~nm}$ as shown in Figure $2 \mathrm{~A}$. Both photocatalysts were able to produce a signature absorbance at $340 \mathrm{~nm}$, with g- $\mathrm{C}_{3} \mathrm{~N}_{4}$ being slower than Pt/g- $\mathrm{C}_{3} \mathrm{~N}_{4}$. After 4.5 hours, the absorbance at $340 \mathrm{~nm}$ for the better of the two catalysts could be interpreted as $\sim 50 \%$ regeneration yield of the 1,4-NADH. However, this information must be used jointly with enzymatic validation to be meaningful. We have determined the total concentration of 1,4$\mathrm{NADH}$ and $\mathrm{NAD}^{+}\left(C_{1,4-\mathrm{NADH}+\mathrm{NAD}}\right)$ using an enzymatic assay. ${ }^{8}$ The cyclic assay utilises two enzymes (lactate dehydrogenase and diaphorase) and two substrates (lactate and MTT) which are dependent on both 1,4-NADH and NAD'. Tracking the formation of formazan (UVVis absorbance at $565 \mathrm{~nm}$ ), the ultimate reduction product, allows for the quantification of $C_{1,4-\mathrm{NADH}+\mathrm{NAD}^{+}}$in a given system (Figure S3). For selective regeneration of $1,4-\mathrm{NADH}$ the $C_{1,4-}$ $\mathrm{NADH}+\mathrm{NAD}^{+}$must remain equal to the starting $C_{0, \mathrm{NAD}^{+}}(0.5 \mathrm{mM})$. Figure $2 \mathrm{~A}$ shows that $C_{1,4-\mathrm{NADH}+\mathrm{NAD}}{ }^{+}$ diminishes with time as the reaction proceeds and becomes effectively zero at 4.5 hours. The $\mathrm{Pt} / \mathrm{g}-\mathrm{C}_{3} \mathrm{~N}_{4}$ catalyst was able to preserve the cofactors slightly better, though still unacceptable. This suggests that without a mediator photocatalytic regeneration over g- $\mathrm{C}_{3} \mathrm{~N}_{4}$ and Pt/g- $\mathrm{C}_{3} \mathrm{~N}_{4}$ cannot produce a significant amount of 1,4-NADH (whose yield is essentially zero) while at the same time losing all of the initial $\operatorname{NAD}^{+}$(i.e., 100\% conversion reached). The $340 \mathrm{~nm}$ absorbance measured, equivalent to a false yield of 50\%, results from 1,6-NADH and/or $\mathrm{NAD}_{2}$ dimers (see Figure 1). These "bad" data show the importance of validating 1,4-NAD(P)H concentration using enzymes.

It is critical to understand what happens to the NAD reactant and produced 1,4-NADH (if any) before a better mediator-free system may be designed. Enzymatic assay data confirmed NAD ${ }^{+}$ is stable for 4.5 hours with $\mathrm{g}-\mathrm{C}_{3} \mathrm{~N}_{4}$ in the dark. We investigated the stability of 1,4-NADH under the same static-batch reaction conditions by starting the reaction with 1,4-NADH instead of 
$\mathrm{NAD}^{+}$. Figure $2 \mathrm{~B}$ shows that the concentration of 1,4-NADH (absorbance at $340 \mathrm{~nm}$ ) decreases with time as soon as the light is switched on. Interestingly when the reaction is performed in a lower triethanolamine (TEOA) concentration the consumption rate of 1,4-NADH is higher, which may be due to TEOA at higher concentration inhibiting access of 1,4-NADH to the photogenerated electrons and holes. With $5 \% \mathrm{v} / \mathrm{v}$ TEOA, nearly half of 1,4-NADH (acting as hole scavenger) is converted to $\mathrm{NAD}^{+}$while the other half is lost/fragmented, whereas with $16 \% \mathrm{v} / \mathrm{v}$ TEOA almost all converted 1,4-NADH is lost as evident by the enzymatic assay data in Figure 2B. We have identified by NMR spectroscopy and negative ion electrospray ionisation mass spectrometry nicotinamide, ADP-ribose, and its derivatives as the ultimate products generated. ${ }^{11}$ The $1,4-\mathrm{NADH}$ consumption rate $(0.189 \mathrm{mM} / \mathrm{h}$, Figure $2 \mathrm{~B})$ is equivalent to that of $\mathrm{NAD}^{+}(0.185 \mathrm{mM} / \mathrm{h}$, Figure $2 \mathrm{~A})$ under identical photocatalytic reaction conditions, suggesting 1,4-NADH will also be subject to fragmentation in a static-batch regeneration system.

Regardless it is clear that the current system (photocatalyst and TEOA in a batch reactor) is unsuitable for mediator-free $\mathrm{NADH}$ regeneration. It is also established that a consecutive reaction mechanism ( $\mathrm{NAD}^{+} \rightarrow$ 1,4-NADH $\rightarrow$ "nicotinamide + ADP ribose + derivatives") is highly plausible. What is not certain is whether a concerted mechanism to the ultimate products is possible, which can be tested by varying contact time. We have therefore designed a flow reactor cell (Figure 3A) which allows controlled contact time of the cofactors with the active surface preventing further reactions of the generated 1,4-NADH, harnessing the advantages of continuous-flow chemistry for limiting unwanted side product formation and allowing for prolonged production. ${ }^{12}$ Figure $3 \mathrm{~B}$ shows the $C_{1,4-\mathrm{NADH}+\mathrm{NAD}}$ as measured by the enzymatic assay and the concentration of NADH products as measured by absorbance at 
$340 \mathrm{~nm}$. Under these conditions, the total cofactor concentration remained constant $\left(=C_{0, N A D^{+}}\right.$, $0.5 \mathrm{mM}$ ) for Pt/g- $\mathrm{C}_{3} \mathrm{~N}_{4}$. i.e., the $340 \mathrm{~nm}$ absorbance is due to 1,4-NADH only. This was verified by an enzymatic assay using diaphorase which detects only $1,4-\mathrm{NADH}$ (Figure S3) $;^{8}$ the concentration obtained matched that calculated from the absorbance at $340 \mathrm{~nm}$. Total steady state single-pass conversion and yield were thus confirmed as $\sim 14 \%$ (Figure $3 \mathrm{C}$ ). The g- $\mathrm{C}_{3} \mathrm{~N}_{4}$ without Pt failed to achieve the same response, suggesting a critical role of Pt in facilitating more efficient and selective interfacial electron/proton transfer to $\mathrm{NAD}^{+}$. The flow cell was further optimised by varying the contact time and we achieved a $100 \%$ single-pass yield of 1,4-NADH continuously over a time period of 5 hours (Figure 3C). In summary, we have highlighted current issues (i.e., the unvalidated yield, the "always-increasing" yield and the missing conversion with accordingly selectivity) hindering progress in $N A D(P) H$ regeneration. Based on our "bad" data, a continuous-flow reactor has been designed that provides a selective and mediator-free approach for photocatalytic cofactor regeneration, opening new opportunities for cooperative catalysis and reactor configurations for future synthesis of chemical and pharmaceutical products. Flow systems may be a promising solution in wider photocatalytic energy conversions where yield/selectivity presents a crucial challenge.

\section{SUPPLEMENTAL INFORMATION}

Supplemental Information includes Experimental Procedures, 1 table, and 4 figures, and can be found with this article online at ...

\section{ACKNOWLEDGMENTS}

UK Catalysis Hub is kindly thanked for resources and support provided via our membership of the UK Catalysis Hub Consortium and funded by EPSRC grant: EP/K014706/2, EP/K014668/1, EP/K014854/1, EP/K014714/1 or EP/M013219/1. W.J., J.W.H.B. and X.W. thank Prof. James 
A. Anderson for contributing to the work before his retirement. X.W., R.F.H. and J.S. thank The Royal Society for initialising (IE150611) and advancing (ICA R1 \180317) this collaboration.

\section{AUTHOR CONTRIBUTIONS}

X.W. conceived and proposed this work with support from R.F.H. and J.S. X.W. and R.F.H. supervised the project. W.J. and J.W.H.B. performed the experiments. J.S. identified the products of NADH conversion. All authors wrote, revised, and approved the manuscript.

\section{REFERENCES}

1. Hambourger, M., Moore, G.F., Kramer, D.M., Gust, D., Moore, A.L., and Moore A.T. (2009). Biology and technology for photochemical fuel production. Chem. Soc. Rev. 38, 25-35.

2. Bornscheuer, U.T., Huisman, G.W., Kazlauskas, R.J., Lutz, S., Moore, J.C., and Robins, K. (2012). Engineering the third wave of biocatalysis. Nature 485, 185-194.

3. Wang, X., Saba, T., Yiu H.H.P., Howe, R.F., Anderson, J.A., and Shi, J. (2017). Cofactor $\mathrm{NAD}(\mathrm{P}) \mathrm{H}$ regeneration inspired by heterogeneous pathways. Chem 2, 621-654.

4. Faber, K. (2018). in Biotransformations in Organic Chemistry, 7th Edition, (Springer), pp. 134.

5. Liu, J., and Antonietti, M. (2013). Bio-inspired NADH regeneration by carbon nitride photocatalysis using diatom templates. Energy Environ. Sci. 6, 1486-1493.

6. Wang, Y., Sun, J., Zhang, H., Zhao, Z., and Liu, W. (2018). Tetra(4carboxyphenyl)porphyrin for efficient cofactor regeneration under visible light and its immobilization. Catal. Sci. Technol. 8, 2578-2587.

7. Burnett, J.W.H., Howe, R.F., and Wang, X. (2020). Cofactor NAD(P)H regeneration: how selective are the reactions? Trends Chem. 2, 488-452.

8. Saba, T., Burnett, J.W.H., Li, J., Kechagiopoulos, P.N., and Wang, X. (2020). A facile analytical method for reliable selectivity examination in cofactor NADH regeneration. Chem. Commum. 56, 1231-1234.

9. Stufano, P., Paris, A.R., and Bocarsly, A. (2017). Photoelectrochemical NADH Regeneration using Pt-Modified p-GaAs Semiconductor Electrodes. ChemElectroChem 4, 1066-1073. 
10. Yuan, M., Kummer, M.J., Milton, R.D., Quah, T., Minteer, S.D. (2019). Efficient NADH regeneration by a redox polymer-immobilized enzymatic system. ACS Catal. 9, 5486-5495.

11. Zhang, S., Shi, J., Chen, Y., Huo, Q., Li, W., Wu, Y., Sun, Y., Zhang, Y., Wang, X., and Jiang, Z. (2020). Unravelling and manipulating of NADH oxidation by photogenerated holes. ACS Catal. 10, 4967-4972.

12. Sambiagio, C., and Noel, T. (2020). Flow photochemistry: shine some light on those tubes! Trends Chem. 2, 92-106. 


\section{FIGURE CAPTIONS}

Figure 1. Schematic Representation of Enzymatic Reduction using 1,4-NADH with in situ Regeneration. Photocatalytic 1,4-NADH regeneration to various products in a batch reactor.

Figure 2. Static-Batch Photocatalysis. (A) Temporal concentration variation of NADH related products (measured by absorbance at $340 \mathrm{~nm}$ ) and total 1,4-NADH + NAD (measured by enzymatic assay ${ }^{8}$ ) for $\mathrm{g}_{-} \mathrm{C}_{3} \mathrm{~N}_{4}$ and $\mathrm{Pt} / \mathrm{g}-\mathrm{C}_{3} \mathrm{~N}_{4}$. Reaction conditions: $0.5 \mathrm{mM} \mathrm{NAD}^{+}$in $0.1 \mathrm{M}$ buffer with $16 \%$ v/v TEOA and 20 mg catalyst. (B) Temporal concentration variation of 1,4$\mathrm{NADH}$ as reactant (measured by absorbance at $340 \mathrm{~nm}$ ). Reaction conditions: $0.5 \mathrm{mM}$ 1,4-

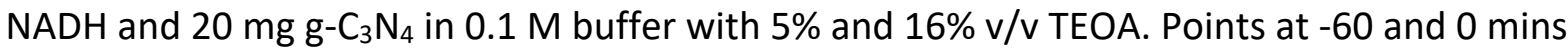
are the reaction mixture in the dark.

Figure 3. Continuous-Flow Photocatalysis. (A) Schematic illustration of the continuous-flow photocatalytic reactor. (B) Temporal concentration variation of NADH related products (measured by absorbance at $340 \mathrm{~nm}$ ) and total 1,4-NADH + NAD (measured by enzymatic assay $^{8}$ ) for $\mathrm{g}-\mathrm{C}_{3} \mathrm{~N}_{4}$ and $\mathrm{Pt} / \mathrm{g}-\mathrm{C}_{3} \mathrm{~N}_{4}$ in a continuous-flow cell. Reaction conditions: $0.5 \mathrm{mM} \mathrm{NAD}^{+}$ in $0.1 \mathrm{M}$ buffer with $16 \% \mathrm{v} / \mathrm{v}$ TEOA, flow rate $2.5 \mathrm{~mL} \mathrm{~h}^{-1}$, with samples for analysis collected in 0.5-h segments. (C) Temporal concentration variation of 1,4-NADH (measured by absorbance at $340 \mathrm{~nm}$ ) and 1,4-NADH (measured by enzymatic assay ${ }^{8}$ ) for Pt/g- $\mathrm{C}_{3} \mathrm{~N}_{4}$ in a continuous-flow cell. Reaction conditions: $0.5,0.05$, and $0.01 \mathrm{mM} \mathrm{NAD}^{+}$in $0.1 \mathrm{M}$ buffer with $16 \% \mathrm{v} / \mathrm{v}$ TEOA, flow rate $2.5 \mathrm{~mL} \mathrm{~h}^{-1}$, with samples for analysis collected in 0.5 -h segments. 